Physical Sciences | Santa Jansone-Popova

\title{
Using diglycolamides to improve rare-earth element separation
}

\begin{abstract}
The family of 17 elements known as the "rare earth
elements" are difficult to

produce economically. Despite this, they are described as "ritical raw materials" because " applications. This means their use is accelerating globally. Separation of these elements is a fundamental challenge, but this does not Popova and her team at Oak Ridge National Laboratory. In recovering praseodymium from neodymium using a diglycolamides, she has bee demonstrating potentially game-changing advances in iquid-liquid separations that demand materials.
\end{abstract}

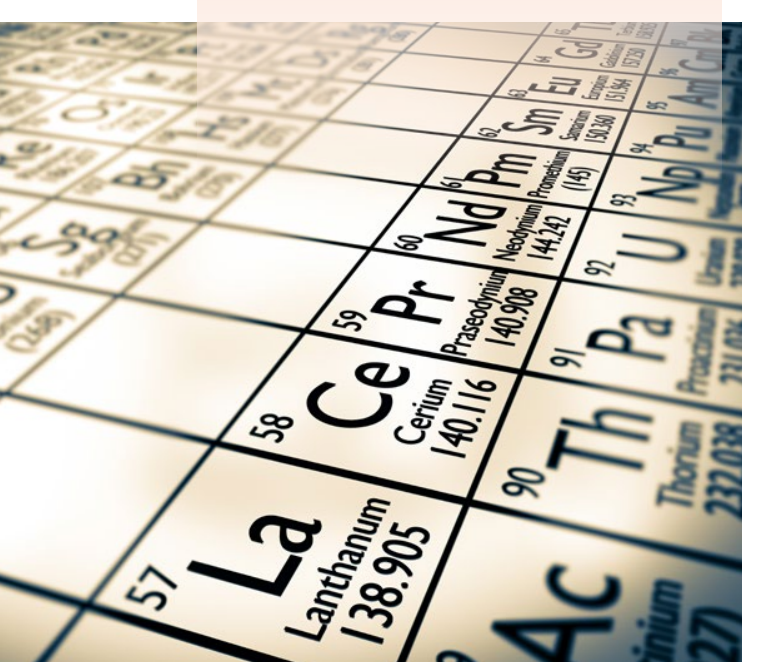

espite their name, the rare earth elements (REEs) are an
abundant group of metallic ements (grouped with the actinides near the bottom of the periodic table) lutetium), scandium, and yttrium. With desirable electronic properties, REEs are exploited in a myriad of technologies and devices ranging from renewable energy applications to military use. Cerium, for example, is an integral component of most catalytic convertors.

\section{LIQUID-LIQUID SEPARATION}

In 2010, a supply crisis arose as escalating This was due to the difficupply issues. REEs. Altho the the diculties in obtaining has its own distinctive electronic properties, collectively the group demonstrates much chemical commonality, which has always presented chemists with enormous challenges, not least in extracting them from each other. Separating adjacent lanthanides $(L n)$ is particularly problematic when they are naturally fused, as they tend to be in mined ores, for example.

When the crisis hit, most countries encouraged further research into liquidaccepted technology for recovering individual REEs. Among other advantages this form of separation can be continuous operated and can lead to substantia gaining more knowledge of this particul methodology might enhance the
Sustainability agenda while contributing to a circular REE economy.

Liquid-liquid extraction is a solvent chnique which uses extractants to transfer metal species between the two immiscible phases. The extractants "hold" metal ions using sulphur and donor groups such as oxygen, metal iond nitrogen. The ligands bind the manner, often in spatially complicated arrangements forming what is known as "complexes". The array of donor atoms directly attached to the central metal is referred to as the first coordination sphere, While a second coordinalion sphere consists of molecules and ions interacting sphere. Although the second sphere has a less directinfluence on the reactivity and chemical properties of a metal complex, they are important in understanding the reactions of the complex as a whole. Liquid-liquid extraction takes metal species from an aqueous medium into an organic solvent, forming combin
separate out into distinct layers.

Key in REE extraction is the selection of the ligands with a right number and arrangement of functional groups as well as the lipophilicity, size and viscosity. Functional groups are groupings of atoms functionality inde that can maintain functionality independenty of other atoms the degree to which the desired metal ions

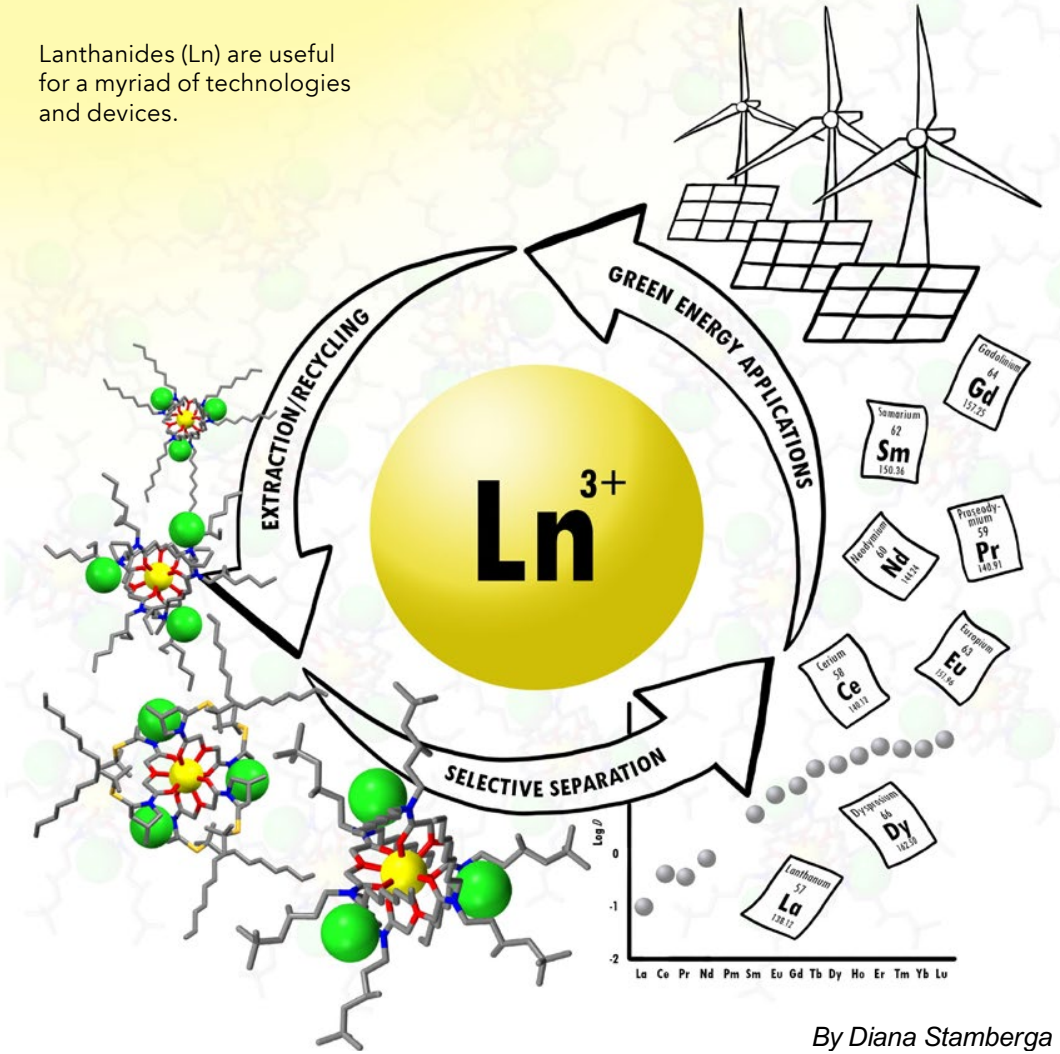

By Diana Stamberga

can be extracted and therefore, extraction Different processes are at play between the organophoshorus acids (acidic extractants) ality of the extractant can be improved and DGAs (neutral extractants). Acidic

Gaining more knowledge of this

ALTERNATIV

CHEMICA

GROUPS

The Chemical

Sciences Division

at Oak Ridge National Laboratory
in Tennessee has particular methodology might enhance

the sustainability agenda while Teen investigating lanthanide and actinide extractants in the hn septo Popova and hecales. Dr Santa Jansoneadding to the expertise, contributing hew insights into the field of solvent extraction. Previously seen as a mature technology, the process typically uses organophosphorus acid as the extractant.

On an industrial scale, the separation is usually accomplished using 2-ethylhexylphosphonic acid mono(2ethylhexyl) ester (EHEHPA for short) where light REEs (lanthanum-gadolinium) get separated from heavy REEs (ytrrium and terbium-lutetium). Ater the introduction of a neutral family of ligands called alky diglycolamides (DGAs) for the separation INL researchers together further with a view th use there a them alternatives to acidic extractants. operate by $\mathrm{pH}$ swing whereas DGAs be lanthanide ions, they have to transfor (coextract) 3 counterions (anions) for each $\mathrm{Ln}^{3+}$ ion in the hydrocarbon solvent as well. This extraction of ions is facilitated at high anion inte aquan and the release of ions back This in This is called the La Chatelier's principle. Acidic extractants do not co pract the mions, since the organophosporus acid acts as a weak acid, i.e. 2 phosphonate molecules form hydrogen bonded dimers, 3 such dimers (each with -1 charge) bind

In DGAs, the metal ion is typically bound to three oxygen atoms within "lipophilic" ligands; these are ligands that dissolve easily in lipids, molecular groups

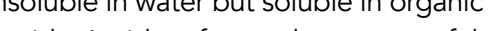
liquids. Amide refers to those parts of the carbonyl nroup (thes atoms are linked to a carbonyl group (these are groups where oxygen atom). By combining different extractants, ions can be surrounded by concentric shell of solvent in a process known as solvation where reorganisation solute molecules into solvation complexes, allowing improved solvent extraction efficiency. Could it be that manipulating the structure of DGAs different chemical environments, more efficient separation of heavy REEs from light REEs could be achieved? Attempting to answer this question extractants in the $L n$ separation process activity relationship of a range of ligands based on DGAs.

CHAINS OF VARYING

\section{IENGTH AND SHAPES}

For the purposes of their studies,
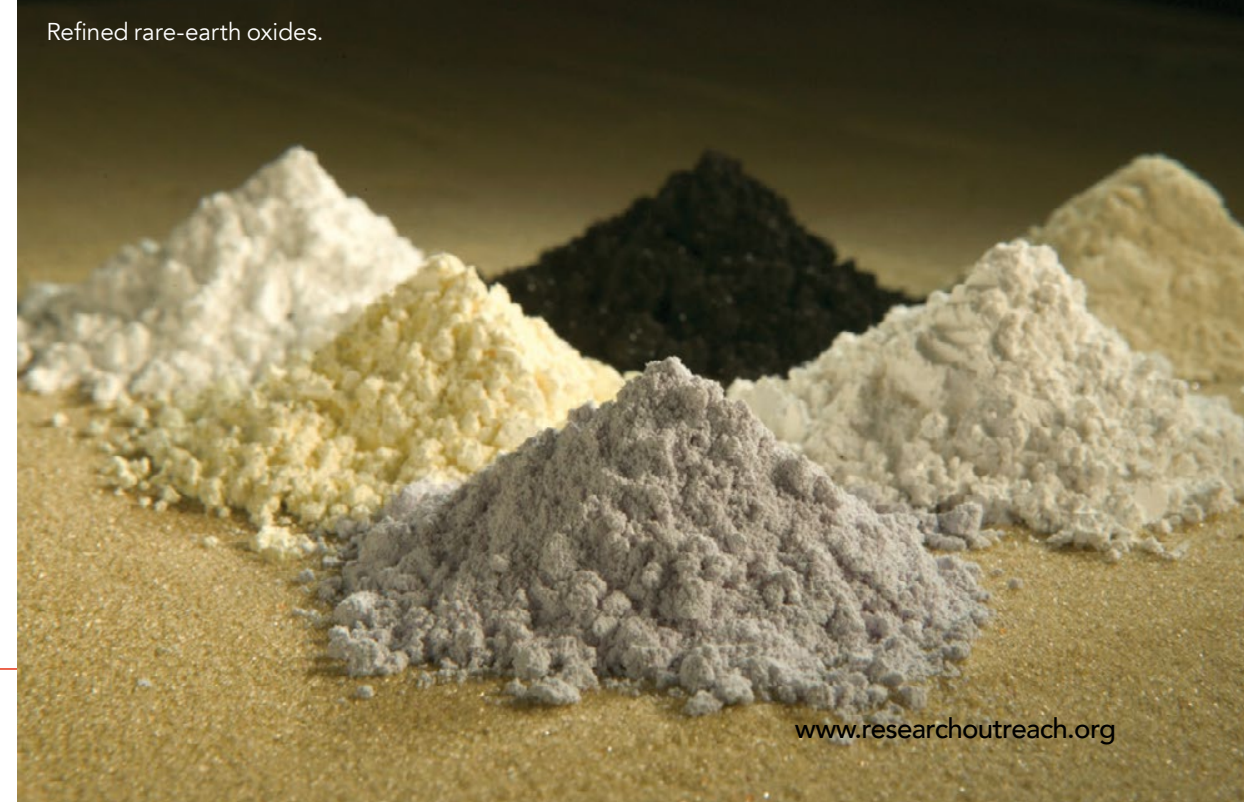


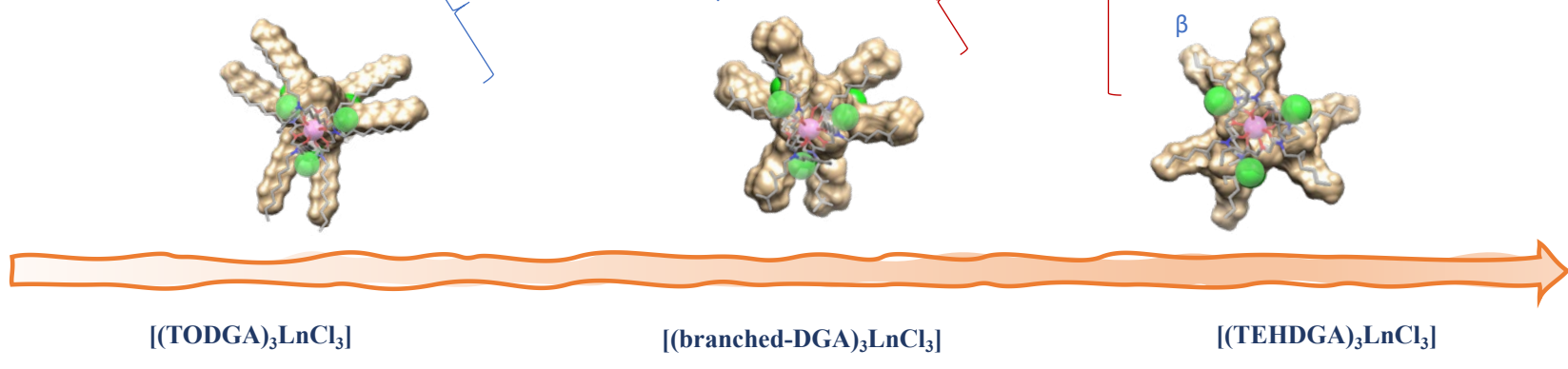

Increase in steric hindrance around the Ln(III) binding site

Improvements in phase-disengagement properties

Increase in Cl-Ln(III) distances

- Decrease in binding strength and selectivity

synthesised a range of functional groups known as alkyls: organic chemicals that extlust . These groups can be and canbon into different structural configurations. For example, their length and shape can be altered, branches created, or linear components transformed into cyclic arrangements, reducing crowding 12 novel DGA-extracting agents were produced, all having differently substituted alkyls. The ability of these compounds to facilitate the separation of 14 lanthanides excluding dioactive promethium) from hydrochloric acid were studied by diglycolamide carbonyl oxygen atoms resulted in dramatic shifts in the selectivity Between the guth and organic phases, differing complex stabilities were experienced faciltating phase-disengagement and intralanthanide selection. Evidence sugges electrostatic interactions beyond the first coordination sphere within a complex dictate extraction behaviour as increasing and decreasing extraction efficiencies were quantified by the researchers.

charge density of ions increases going from $\mathrm{La}^{3+}$ to $\mathrm{Lu}^{3+}$

The researchers also reported that by shortening alkyl substituents or by placing mide functional gites further away from extraction process. Some of the rationale for this is placed on the need for strong binding between the extractants and metal ions. In other findings, less steric hindrance in the alkyl chain attached to the amide groups demonstrated etter extraction

These findings are encouraging and directly ability than their contribute to the possibility of greater counterparts while efficiencies in separation chemistry. $\quad \begin{aligned} & \text { decreased extraction } \\ & \text { efficiencies may be } \\ & \text { due to increased }\end{aligned}$ due to increased

extractants in hydrocarbon solvent. It was found that as ligand size increased, crowding around the metal-ion binding

\section{OBSERVING STRUCTURE-}

PERFORMANCE RELATIONSHIPS Adopting a structure-performance most extensively studied DGAs (these have the acronyms TODGA, TEHDGA and DMDODGA), the 12 varied extractants were designed to study the interplay of "steric" and electronic effects (where steric is the spatial arrangement of atoms in a molecule) that control ra earth element extraction efficiency.

Probing the structure of complexes that were formed using $\mathrm{X}$-ray spectroscopy, team made some stark discoveries, observing that the structure of the myl chains plays a key role in the metal ion extraction performance. Just redraction of lanthanides was markedy observed when these spacious groups were placed too close to the DGA oxygen atoms.

The team observed better extraction from lanthanum going through the series to lutetium, the decrease in ion radius seemingly increasing extraction tross the series and betwe adjacent lanthanides (in the periodic table). It is thought that the lipophilic ligands leverage incremental change in ionic radius brought about by lanthanid contraction. Exponential improvement in extraction was observed across the lanthanide series and thought to be underpinned by the ability of extractant during the separation prochs as site interfering with the efficient secondany coordination spher

\section{ADVANCING DEVELOPMENTS} All of these findings are encouraging and greater efficiencies in separation chemisty. If economic extraction from source is to be enabled, this will be key to addressing the need for critical materials. The work of Dr Jansone-Popova et al. provides innovative ideas as to how improved separations of light lanthanides could be obtained. In ascertaining how subtle steric and electronic changes markedly from hy extractant performance from hydrochloric acid media, they redvention implementation of next-generation

Dr Santa Jansone-Popova

E: jansonepopos@ornl.gov $\quad$ T: +1 713-231-4865 T: +1 865-576-2048
W: https://www_ornl.gov/news/santa-jansone-popova-chemistry-design

\section{Research Objectives}

DrSanta Jansone-Popova and colleagues are researching quid-liquid separations of rare earth elements.

\section{Detail}

\section{Address}

Oak Ridge National Laboratory

O. Box 2000 MS

Oak Ridge, TN 37831

Dr Santa Jansone-Popova completed her undergraduate studies at the University of Latvia. In 2009, she moved to Texas to pursue graduate studies at the University of Houston. After graduation in 2014, she joined the Chemical Separations group in Chemical Sciences Division at Oak Ridge National Laboratory.

Funding

The Critical Materials Institute, an Energy Innovation Hub funded by the U.S. DOE, Office of Energy Efficiency and
Renewable Energy, Advanced Manufacturing Office.

\section{Collaborators}

Diana Stamberga, Mary R. Healy, Vyacheslav S. Bryantsev, Camille Albisser, Yana Karslyan, Benjamin Reinhart, Alena Paulenova, Mac Foster, Ilja Popovs, Bruce A. Moyer, Kevin Lyon

\section{OAK RIDGE} National Laboratory

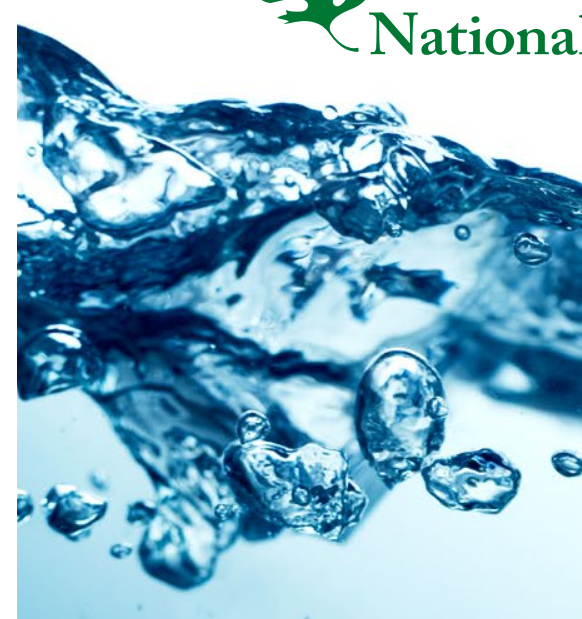

(2) 0
References

Špadina, M., Bohinc, K., Zemb, T. and Durêeche, J.F. (2019) Synergistic Solvent Extraction is Driven by Entropy. ACS Nano, 13(12), 13745. Available at: https://doi.org/10.1021/ acsnano.9607605

Cheisson, T. and Schelter, E. J. (2019) Rare earth elements: Mendeleev's bane, Modem mavels. Science, 363(6426); 48

Sholl, D. S. and Lively, R. P. (2016) Seven chemical separations to change the world. Nature, 532, 435. Available at:

https://www.nature.com/news/seven-chemical-separations-tochange-the-world-1.19799

\section{Personal Response}

Is there the possibility of exploring another ligand

II Developing processes that result in more efficient separation of adjacent $L$ (IIII) is the key. This will lead to reduced processing costs and improved waste management. The neutral extractants (diglycolamides)
offer better selectivity than the acidic extractants based on organophosphorus compounds. We are actively
exploring other ligand families that show more than exploring other ligand families that show more than 2-fold increase in selectively separating the adjacent lanthanides. Given the ever-growing demand tor critical
materials, we expect the emergence of more efficient
separation processes in the years to come.

What more could be done to significantly reduce

processing costs?

Reduced chemical consumption and their recycling smaller number of separation stages, and use of higher efficiency - are the main drivers to reduce with processing costs. This is a challenging task that motivates chemists and engineers to work together to develop
better separation processes.
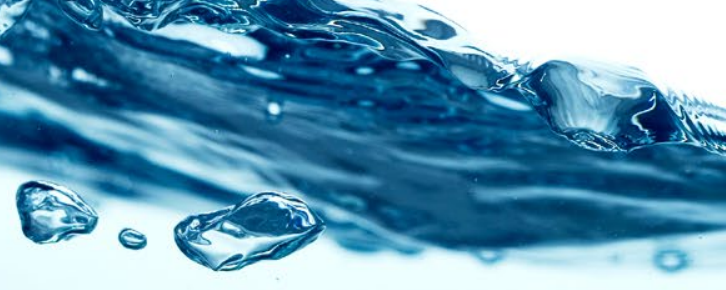

www.researchoutreach.org 